\title{
KIS: Synaptic Plasticity’s Missing Molecular Link?
}

\author{
(D) Jon Brudvig ${ }^{1,2}$ and Jacob Cain ${ }^{2}$ \\ ${ }^{1}$ Basic Biomedical Sciences, Sanford School of Medicine, University of South Dakota, Vermillion, South Dakota 57059, and 2Children's Health Research \\ Center, Sanford Research, Sioux Falls, South Dakota 57104 \\ Review of Pedraza et al.
}

Long-term potentiation (LTP) is a well characterized form of synaptic plasticity. In NMDA receptor-dependent LTP, presynaptic activity coincident with postsynaptic depolarization results in the influx of calcium ions through NMDA receptors in the membranes of dendritic spines. This calcium influx has multiple downstream effects mediated by interactions with a number of intracellular protein partners, including $\mathrm{Ca}^{2+} /$ calmodulin-dependent protein kinases and PKC. The end result, in the case of LTP, is synapse reinforcement through cytoskeletal remodeling, AMPA receptor trafficking and modification, and local protein synthesis (Derkach et al., 2007). The intermediates between $\mathrm{Ca}^{2+}$-activated kinases and structural changes, however, remain largely unknown (Purves, 2012).

The ultimate outcome of this process is the coupling of synaptic activity with synaptic structure; spines that receive enough input will be maintained and strengthened, while those that do not are lost. This associative characteristic underlies the molecular basis of learning and memory, and is largely dependent on the local nature of the synaptic changes. One neuron can have hundreds to thousands of dendritic spines, and each can be regulated locally by activity dependent mechanisms,

Received Dec. 15, 2014; revised Jan. 9, 2015; accepted Jan. 14, 2015. We thank Dr. Jill Weimer and Derek Timm for their editorial assistance. Correspondence should be addressed to Jon Brudvig, Sanford Research, 2301 East $60^{\text {th }}$ Street North, Sioux Falls, SD 57104. E-mail: Jon.Brudvig@SanfordHealth.org.

DOI:10.1523/JNEUROSCI.5082-14.2015

Copyright $\odot 2015$ the authors $\quad 0270-6474 / 15 / 352839-03 \$ 15.00 / 0$ cytoskeletal dynamics, and protein synthesis. Translational control is achieved through structures called messenger ribonucleoprotein (mRNP) granules (Buchan, 2014). In neurons, synaptic mRNAs are transcribed and processed in the nucleus and packaged, along with various nontranslating mRNAs, regulatory proteins, and ribonucleoproteins, into mRNP granules. These structures facilitate the selective transport, localization, and translation of mRNAs while preventing their degradation (Buchan, 2014). This process brings mRNAs to their targeted locations, and allows them to remain latent until activated by the appropriate signals. Pedraza et al. (2014) have established an important function for KIS, a protein kinase with a unique RNA binding domain, in this mRNA regulatory process within dendritic spines, setting the stage for further exploration into the role of KIS in synaptic plasticity.

To study the function of KIS in spine development in mature neurons, Pedraza et al. (2014) knocked down KIS in cultured hippocampal neurons with a lentiviral construct coexpressing GFP and a shRNA targeted against KIS (shKIS). KIS knockdown drastically reduced dendritic arborization and altered dendritic spine morphology. While spine density was similar, total spine area was significantly reduced as a result of altered shape. shKIS spines took on a thin, filopodia-like shape, with reduced or absent heads, while control spines were largely mushroom-shaped, with wide, bulbous heads with more surface area for synaptic contact (Pedraza et al. 2014, their Fig. 1). These morphological changes suggest cytoskeletal alterations. In vitro, KIS has been shown to interact with several important cytoskeletal regulators, including stathmin, which modulates tubulin dynamics (Maucuer et al., 1997), as well as mRNAs important for cytoskeletal dynamics.

The same lab previously demonstrated that KIS colocalizes with $\beta$-actin mRNA in cortical neurons (Cambray et al., 2009), and $\beta$-actin plays an important role in spine formation and maturation (Hotulainen and Hoogenraad, 2010). Thus, Pedraza et al. (2014) explored whether KIS influences $\beta$-actin polymers. While healthy control neurons displayed robust f-actin staining (as labeled by phalloidin), neurons with reduced KIS levels had diminished postsynaptic polymerization of $\beta$-actin in their dendritic spines (Pedraza et al., 2014, their Fig. 2). It is likely that this decrease contributes to the morphological changes observed in shKIS spines, but the complete absence of mature, bulbous spine heads strongly suggests alterations in other proteins specific to the postsynaptic density region. Indeed, levels of the AMPA receptor subunits GluR1 and GluR2, as well as the postsynaptic density scaffolding protein PSD-95, were significantly reduced in shKIS neurons. For AMPA receptor subunits, both total and surface levels were reduced (by $60 \%$ for GluR1 and 20\% for GluR2) (Pedraza et al., 2014, their Fig. 4), suggesting a deficiency in expression rather than abnormal receptor cycling in the spine. 
Such drastic changes in glutamate receptors, PSD-95 levels, and spine morphology should have a great impact on excitatory signaling. To test this, the authors performed whole-cell recordings of miniature EPSCs (mEPSCs) in shKIS neurons. As expected from the observed alterations, mEPSC currents had a 30\% reduction in amplitude, reflecting a deficit in postsynaptic receptiveness. Additionally, there was a significant reduction in frequency, as measured by interevent interval, which likely reflects a loss of functional synapses (Pedraza et al., 2014, their Fig. 3). These results demonstrate the functional importance of KIS in the synapse, where even small perturbations in activity can have dramatic effects.

The next step was to examine how KIS exerts its influence on postsynaptic protein levels. This team previously demonstrated that KIS stimulated $\beta$-actin mRNA translation through a direct or indirect interaction with the $3^{\prime}$ untranslated region (UTR) (Cambray et al., 2009). Experiments in the present study determined that KIS similarly interacts with the 3' UTR region of GluR2, stimulating translation. Cytoplasmic polyadenylation element binding protein 3 (CPEB3) was identified as a necessary partner in this interaction; when the authors knocked down CPEB3 along with KIS, GluR1 and GluR2 levels were restored (Pedraza et al., 2014, their Fig. 5). Still, the nature of these interactions remained unknown. Is KIS binding CPEB3 directly, or a shared substrate mRNA?

CPEB3 was previously shown to inhibit translation of AMPA receptor mRNAs by binding the $3^{\prime}$ UTR and preventing polyadenylation (Huang et al., 2006; Pavlopoulos et al., 2011). Pedraza et al. (2014) hypothesized that KIS may be alleviating this inhibition through a direct interaction with CPEB3, stimulating polyadenylation and translation. Several results support this notion: KIS was demonstrated to interact with CPEB3 in HEK293T cells in an RNase-resistant complex. Additionally, CPEB3 was demonstrated to bind the 3' UTR of the GluR2 transcript and reduce its polyadenylation level. In the presence of KIS, CPEB3GluR2 3' UTR binding was significantly reduced, and GluR2 3' UTR polyadenylation was restored (Pedraza et al., 2014, their Fig. 6). Still, determining the precise mechanics of this interaction will require more work. Is KIS blocking the RNAbinding site of CPEB3, preempting the inhibition of polyadenylation and translation, or is this an allosteric interaction that releases $\mathrm{CPEB} 3$ from mRNAs in response to synaptic activity? KIS is known to be a substrate of several other kinases found in dendritic spines. Does phosphorylation regulate the ability of KIS to alleviate CPEB3-mediated translational inhibition?

Pedraza et al. (2014) have demonstrated that KIS interacts with CPEB3 to regulate $3^{\prime}$ UTR-mediated stimulation of translation for GluR2. Furthermore, KIS stimulates translation of GluR1 and PSD-95 and enhances $\beta$-actin polymerization in dendritic spines. These results provide a convincing explanation for the spine morphological defects observed in the absence of KIS: with reduced levels of several of the key proteins necessary for dendritic spine maturity, spines do not develop beyond the initial stages, and retain filopodia-like shape. This also demonstrates a possible function for KIS in synaptic plasticity including processes like LTP, but more work is required to solidify this role.

KIS is a particularly attractive protein for the study of dendritic plasticity if it is indeed latent in the spine until activated in response to synaptic activity. Thus far, however, the effects of KIS on spine remodeling have only been determined on a cell-wide level with limited temporal resolution. KIS has been shown to shuttle between the nucleus and cytoplasm, interacting with various substrates at both sites (Francone et al., 2010). The results of Pedraza et al.'s (2014) study do not exclude the possibility that KIS influences translation by regulating transport of mRNA granules to the dendritic spine, where it inhibits CPEB3-mRNA interactions. Studies demonstrating precisely where KIS and substrate mRNAs colocalize could help determine whether and where KIS is interacting with mRNAs important in dendritic spine development. Questions regarding temporal regulation will need to be addressed as well, in part with experiments testing the effect of phosphorylation on KIS activity.

Another important next step will be to determine whether KIS is activated in response to presynaptic and postsynaptic cues. If KIS stimulates translation of GluR1, GluR2, PSD-95, and $\beta$-actin in response to synaptic activity, it could be a key regulator of processes like LTP. It is unknown how KIS knockdown affects these processes. Further studies should examine how the presence or absence of KIS affects LTP in hippocampal slices or cultures, and should investigate potential interactions that could transduce synaptic activity into KIS activity. CaMKII is a prime candidate for such an interaction. It is abundant in synapses exhibiting LTP, its deletion precludes LTP, and, while its downstream targets include AMPA receptors, the intermediates in this pathway remain unknown (Purves, 2012). Notably, KIS has been experimentally determined to be a physiologically relevant substrate for CaMKII (Newman et al., 2013). This interaction surely deserves additional attention in light of Pedraza et al.'s (2014) recent findings.

If KIS is determined to interact with CaMKII in plasticity-related processes, it will contribute greatly toward a robust understanding of the molecular substrates of synaptic plasticity. CaMKII activation was recently demonstrated to locally influence spine volume following synaptic activity through interactions with the Rho GTPases Cdc42 and RhoA (Murakoshi et al., 2011), both of which are known to impact actin dynamics within the spine. Interestingly, Cdc42 is also regulated by Tnk2, which is an upstream kinase that phosphorylates KIS (Newman et al., 2013). Presynaptically, a similar CaMKIIcontrolled network appears to be operating, with CaMKII regulating a variety of plasticity-related substrates, including synapsin-1 (Menegon et al., 2002) and KIS, which also interact with each other.

The demonstrated roles for neuronal KIS are rapidly increasing. Pedraza et al. (2014) have demonstrated that KIS is important for dendritic spine development and maintenance, as well as non-spinerelated processes including dendritic arborization. KIS has also been shown to interact with proteins involved in neuropeptide production (Francone et al., 2010), synaptic vesicle recycling (Menegon et al., 2002), and axonogenesis (Menegon et al., 2002). Any protein with such widespread influence on neuronal function warrants further study.

\section{References}

Buchan JR (2014) mRNP granules: assembly, function, and connections with disease. RNA Biol 11:1019-1030. CrossRef Medline

Cambray S, Pedraza N, Rafel M, Garí E, Aldea M, Gallego C (2009) Protein kinase KIS localizes to RNA granules and enhances local translation. Mol Cell Biol 29:726-735. CrossRef Medline

Derkach VA, Oh MC, Guire ES, Soderling TR (2007) Regulatory mechanisms of AMPA receptors in synaptic plasticity. Nat Rev Neurosci 8:101-113. CrossRef Medline

Francone VP, Ifrim MF, Rajagopal C, Leddy CJ, Wang Y, Carson JH, Mains RE, Eipper BA (2010) Signaling from the secretory granule to the nucleus: Uhmk1 and PAM. Mol Endocrinol 24:1543-1558. CrossRef Medline 
Hotulainen P, Hoogenraad CC (2010) Actin in dendritic spines: connecting dynamics to function. J Cell Biol 189:619-629. CrossRef Medline

Huang YS, Kan MC, Lin CL, Richter JD (2006) CPEB3 and CPEB4 in neurons: analysis of RNA-binding specificity and translational control of AMPA receptor GluR2 mRNA. EMBO J 25:4865-4876. CrossRef Medline

Maucuer A, Ozon S, Manceau V, Gavet O, Lawler S, Curmi P, Sobel A (1997) KIS is a protein kinase with an RNA recognition motif. J Biol Chem 272:23151-23156. CrossRef Medline

Menegon A, Verderio C, Leoni C, Benfenati F, Czernik AJ, Greengard P, Matteoli M, Val- torta F (2002) Spatial and temporal regulation of $\mathrm{Ca} 2+/$ calmodulin-dependent protein kinase II activity in developing neurons. J Neurosci 22:7016-7026. Medline

Murakoshi H, Wang H, Yasuda R (2011) Local, persistent activation of Rho GTPases during plasticity of single dendritic spines. Nature 472:100-104. CrossRef Medline

Newman RH, Hu J, Rho HS, Xie Z, Woodard C, Neiswinger J, Cooper C, Shirley M, Clark HM, Hu S, Hwang W, Jeong JS, Wu G, Lin J, Gao X, Ni Q, Goel R, Xia S, Ji H, Dalby KN, et al. (2013) Construction of human activitybased phosphorylation networks. Mol Syst Biol 9:655. CrossRef Medline
Pavlopoulos E, Trifilieff P, Chevaleyre V, Fioriti L, Zairis S, Pagano A, Malleret G, Kandel ER (2011) Neuralized1 activates CPEB3: a function for nonproteolytic ubiquitin in synaptic plasticity and memory storage. Cell 147: 1369-1383. CrossRef Medline

Pedraza N, Ortiz R, Cornadó A, Llobet A, Aldea M, Gallego C (2014) KIS, a kinase associated with microtubule regulators, enhances translation of AMPA receptors and stimulates dendritic spine remodeling. J Neurosci 34:13988-13997. CrossRef Medline

Purves D (2012) Neuroscience, 5th edition. Sunderland, MA: Sinauer Associates. 